\title{
A Study of Cyto-Histological Correlation in the Diagnosis of Thyroid Swelling
}

\author{
Rupam Borgohain ${ }^{1}$, Ranjeet Kumar Lal ${ }^{2}$, Pritam Chatterjee ${ }^{2}$, Nency Brahma ${ }^{2}$, \\ Swagata Khanna ${ }^{3}$ \\ ${ }^{1}$ (Assistant Professor, Department of ENT, Gauhati Medical College \& Hospital, Guwahati, Assam, India) \\ 2 (Junior Residents, Department of ENT, Gauhati Medical College \& Hospital, Guwahati, Assam, India) \\ ${ }^{3}$ (Professor \& Head of Dept., Department of ENT, Gauhati Medical College \& Hospital, Assam, India)
}

\begin{abstract}
There is some "grey zone" in thyroid cytology where the diagnostic efficacy declines sharply rendering it difficult to categorize the lesions resulting in discrepancy. So, a cross-sectional study was conducted to determine the accuracy of FNAC in diagnosis of thyroid swelling and to assess the correlation between preoperative cytological diagnosis and post operative histopathological diagnosis. It was observed that FNAC is reliable, safe and accurate method as a first line of evaluation in thyroid swelling before the surgery. FNAC is more sensitive in detecting thyroid gland malignancy and therefore histopathological analysis still remains essential for final diagnosis.
\end{abstract}

Key words: Thyroid swelling, FNAC, Histopathology.

\section{Introduction}

Thyroid swelling remains a problem of enormous magnitude all over the world. The problem in clinical practice is to distinguish reliably the few malignant tumors from the many harmless nodules so that a definitive preoperative tissue diagnosis of the malignancy allows planning of appropriate surgery and relevant patient counseling. The prevalence of thyroid swelling ranges from $4 \%$ to $10 \%$ in the general adult population and from $0.2 \%$ to $1.2 \%$ in children ${ }^{1}$. The majority of clinically diagnosed thyroid swelling are non-neoplastic; only $5 \%$ to $30 \%$ are malignant and require surgical intervention ${ }^{2}$ In India, thyroid cancer comprises of $1 \%$ of all head and neck cancers.

Fine needle aspiration cytology (FNAC), being simple, readily available, reliable, time saving, minimally invasive, cost effective, having high sensitivity and specificity have been applied routinely as a useful and indispensible method to diagnose thyroid lesions. FNAC has allowed a dramatic decrease in unnecessary surgeries without thyroid nodular disease, enhancing the percentage of malignant operated nodules over $50 \%$. It is relied upon to distinguish benign from neoplastic or malignant thyroid nodules, thus, influencing therapeutic decisions. However, FNAC has its limitations. Histopathological examination of surgically excised thyroid swelling is the most accurate way to determine the pathology.

\section{Aims \& Objectives}

The present study is undertaken to evaluate the accuracy of thyroid FNA and its correlation with histopathological examination.

\section{Materials And Methods}

A cross sectional study was done in the department of ENT - Head and Neck Surgery, from January 2012 to December 2013 (2 years). All patients were evaluated by thorough clinical examination followed by routine investigations, thyroid function tests, FNAC and histopathological examination. The cytology reports were compared with the histological diagnosis. Sensitivity, specificity, accuracy, positive predictive value and negative predictive value were calculated.

Inclusion criteria- Those patients presenting with thyroid swelling, who underwent FNAC, thyroid surgery and histopathological examination within the study period were included in the study.

Exclusion criteria- All the cases of thyroiditis were excluded. Those patients having FNAC done but did not have thyroid surgery were excluded.

\section{Results}

In this series of 122 thyroid swellings, patients were grouped in age groups of 0-20; 21-40; 41-60 and 61-80 years \& patients in each age group were $9 \% ; 50 \% ; 37 \%$ and $4 \%$ respectively. Most of the patients were age group of 21-40 years. Female were higher in frequency $(n=109 ; 89.34 \%)$ than male $(n=13 ; 10.66 \%)$. 
On FNAC, 85 cases (70\%) were non-neoplastic and 37 cases $(30 \%)$ were neoplastic disease. Among the nonneoplastic swellings, colloid goiter was most common with 50 cases $(41 \%)$, followed by multi-nodular goiter (MNG) with 18 cases (15\%) and nodular goiter which was 16 cases (13\%). Among the neoplastic thyroid swelling, papillary carcinoma was the most common with 17 cases (14\%).

After post operative histopathological examination, 92 cases $(75.40 \%)$ were found non-neoplastic and 30 cases $(24.50 \%)$ were found neoplastic. Among the ninety-two cases of non-neoplastic thyroid swelling, colloid goiter was 50 cases (41\%), followed by nodular goiter with 22 cases (18\%), MNG with 9 cases $(7 \%)$ cases, hashimotos thyroiditis with 6 cases $(5 \%)$, benign haemorrhagic cyst with 3 cases $(2.4 \%)$, and one case each of benign colloid cyst and chronic thyroiditis. Among the neoplastic thyroid swelling, papillary carcinoma was the most common with 12 cases $(10 \%)$, followed by follicular adenoma with 9 cases, follicular carcinoma 5 cases and medullary carcinoma with 4 cases.

Table 1: Variation of diagnosis between FNAC and Histopathology in thyroid swelling (n=122).

\begin{tabular}{|l|l|l|l|}
\hline \multirow{4}{*}{ Non-neoplastic } & Diagnosis & FNAC & Histopathology \\
\cline { 2 - 4 } & Colloid goitre & 50 & 50 \\
\cline { 2 - 4 } & MNG & 18 & 9 \\
\cline { 2 - 4 } & Nodular goiter (STN) & 16 & 22 \\
\cline { 2 - 4 } & Hashimotos thyroiditis & - & 6 \\
\cline { 2 - 4 } & Chronic thyroiditis & - & 1 \\
\cline { 2 - 4 } & Benign haemorrhagic cyst & - & 3 \\
\cline { 2 - 4 } & Benign follicular disease & 1 & - \\
\cline { 2 - 4 } & Benign colloid cyst & - & 1 \\
\cline { 2 - 4 } & Follicular neoplasm & 10 & - \\
\cline { 2 - 4 } & Follicular adenoma & 9 & 9 \\
\cline { 2 - 4 } & Follicular carcinoma & - & 5 \\
\cline { 2 - 3 } & Papillary carcinoma & 17 & 12 \\
\cline { 2 - 3 } & Medullary carcinoma & - & 4 \\
\cline { 2 - 3 } & Hurtle cell neoplasm & 1 & - \\
\hline Total (n) & & 122 & 122 \\
\hline
\end{tabular}

On comparing the finding of FNAC and post operative histopathology examination, we see:

For non-neoplastic cases, chi square test $\left(\mathrm{X}^{2}\right)=11.120$, with degree of freedom (d.f.) $=3$, we get probability $(p)<0.05$, i.e. the two tests gives us significantly different results.

For neoplastic cases, $X^{2}=4.69$, with degree of freedom (d.f.) 2, we get $p$ value $>0.05$, i.e. the results are not significantly different for the two tests.

Two cases of nodular goitre in FNAC was diagnosed as Papillary carcinoma in HPE, whereas out of 4 cases of papillary carcinoma in FNAC came out to be 2 cases of hashimotos thyroiditis, one case of chronic thyroiditis and one case of colloid goitre

Out of FNAC diagnosed 10 cases follicular neoplasm, 5 cases $(50 \%)$ came out to be follicular adenoma and 5 cases $(50 \%)$ came out to be follicular carcinoma.

Out of 122 cases, 50 diagnosed as colloid goiter, 22 cases as nodular goitre, 9 cases as MNG, 6 cases as hashimotos thyroiditis, 3 cases as benign haemorrhagic cyst, 1 case each as chronic thyroiditis and benign colloid cyst; 12 cases as papillary carcinoma, 9 cases as follicular adenoma , 5 cases as follicular carcinoma and 4 cases as medullary carcinoma.

The cytological diagnoses were then compared with available histopathological diagnoses. Cyto-histological concordance was obtained in 89 cases $(72 \%)$.

Table 2: the accuracy of diagnostic test of FNAC in thyroid swelling.

\begin{tabular}{|c|c|c|}
\hline Test (FNAC) & Malignant in histopathology & Benign in histopathology \\
\hline Positive test & True positive (TP) & False positive (FP) \\
& 23 & 12 \\
\hline Negative test & False negative (FN) & True negative (TN) \\
& 5 & 79 \\
\hline
\end{tabular}

Sensitivity shows the portion of the patients having malignant thyroid disease and positive cytological diagnosis on FNAC, which is found to be $82.14 \%$.

Specificity shows the portion of the patients with non-malignant thyroid disease and positive cytological diagnosis, which was found to be $86.81 \%$ :

Accuracy is the portion of the correct results, true positive and true negative in relation to all cases studied is found to be $83.60 \%$ in our study.

Positive predictive value (PPV) is the probability of having malignant thyroid disease following a positive FNAC finding and is found to be $65.71 \%$ here. 
Negative predictive value ( NPV) is the probability of not having malignant thyroid disease following a negative FNAC finding and is found to be $94.04 \%$.
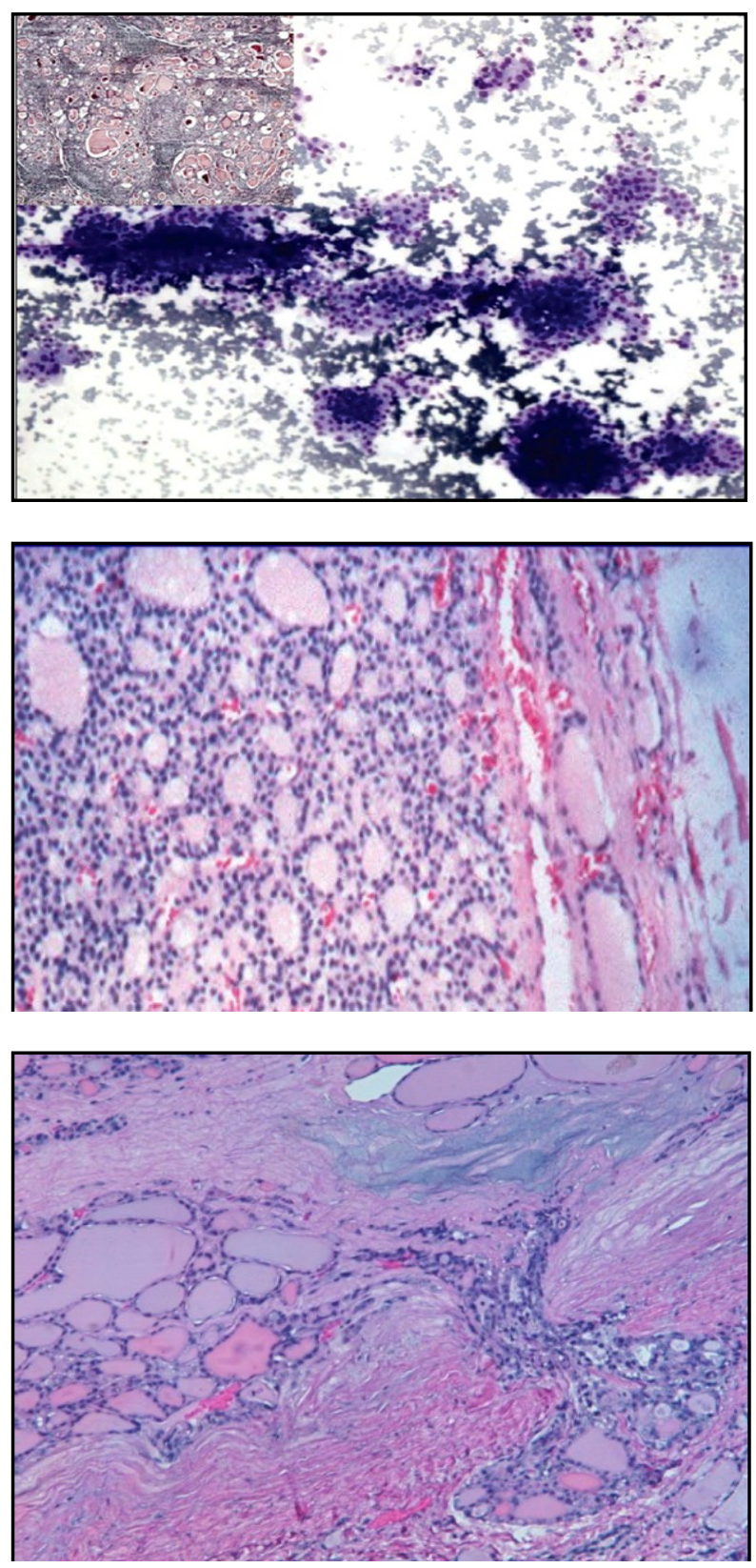

Fig 1 : Too much stress on papillary architecture and intranuclear inclusions led to erroneous diagnosis of papillary carcinoma on cytology (MGG, x100). Inset showing the follow up histology of Hashimoto's thyroiditis ( Inset; $\mathrm{H}$ and $\mathrm{E}, \mathrm{X}$ 40)

Fig 2: Follicular and microfollicular adenoma shows microfollicles, is sharply circumscribed by a delicate even fibrous capsule, no invasion of the capsule or blood vessels by the tumour (Inset; $\mathrm{H}$ and $\mathrm{E}, \mathrm{X} 40$ )
Fig 3: Follicular carcinoma showing angioinvasion (Inset; $\mathrm{H}$ and $\mathrm{E}, \mathrm{X} 40$ )

\section{Discussion}

FNAC contributes significantly to the preoperative investigation in patients with thyroid swelling but despite its well recognized value there are limitations to the technique. The reported pitfalls are those related to specimen adequacy, ${ }^{4,5}$ sampling techniques, the skill of the aspirator performing the aspirations, the experience of the cytopathologist interpreting the aspirate and overlapping cytological features between benign and malignant follicular neoplasms and inadequate, indeterminate FNA. One major limitation of thyroid cytology is its inability to distinguish between follicular adenoma from follicular carcinoma. ${ }^{5,67,8}$ this diagnosis require detail histological examination for vascular or capsular invasion and cannot be reliably made on routine FNAC specimens. ${ }^{9,10,11,12}$ hence, follicular neoplasm (lesion) is given as diagnosis in FNAC.

In the present study of 122 cases, majority of cases were seen in the age group of $21-40$ years $(50 \%)$. Female were higher in frequency $(89.34 \%)$ than male $(10.66 \%)$. The youngest patient of this series was a a girl of 11 years with colloid goitre and the oldest patient was a man of 70 years, a case of follicular carcinoma.

In this study, FNAC showed 30\% neoplastic cases. Among neoplastic thyroid swelling papillary carcinoma was the most common with $14 \%$, followed by follicular neoplasm $8.19 \%$. Carcinoma of the thyroid is the most common malignancy of endocrine system comprising .6\% and 1.6 of all cases of malignant neoplasm 
in men and women respectively (Aravindan,2006).In this study, among non neoplastic thyroid swelling (70\%), colloid goitre was common $41 \%$, followed by MNG which was $15 \%$ and nodular goitre with $13 \%$. The study reported by Md. Shafiqul Islam showed 78\% non-neoplastic and 22\% neoplastic.( papillary carcinoma , $15.56 \%$ and follicular lesion $3.3 \%)^{16}$

While comparing FNAC with histopathological examination (HPE) in the present study, it was found that $41 \%$ cases of colloid goitre was diagnosed as colloid goitre in HPE; $15 \%$ of MNG proved to be $7.37 \%$ in HPE. Out of 17 cases of papillary carcinoma, on histopathology 3 cases came out to be medullary carcinoma, 2 cases came out to be hashimotos thyroiditis and 1 case each of chronic thyroiditis and colloid goitre. Whereas out of 12 cases of papillary carcinoma on HPE two cases were previously diagnosed as nodular goitre.

In this study, out of FNAC diagnosed 10 cases follicular neoplasm; $50 \%$ came out to be follicular adenoma and 50\% came out to be follicular carcinoma. WC. Faquin, 2009 reported $15-30 \%$ of FNA diagnosed follicular neoplasm were actually carcinomas; remaining $70-85 \%$ were benign. ${ }^{17}$ Baloch $\mathrm{ZW}, 2002$ observed that the diagnosis " follicular neoplasm" is indeterminate; $70 \%$ are benign. ${ }^{18}$

Cytohistological correlation was present in $72 \%$ of cases in this study. Monisha et al., 2011 reported cytological correlation in $80 \%$ of cases. ${ }^{19}$ Pinky Pandey et al. 2012 also reported cytohistological concordance in $80.28 \%$ of the cases. ${ }^{20}$

In the present study we found sensitivity by FNAC to be $82.14 \%$, the specificity was $86.81 \%$, the PPV was $83.60 \%$ and NPV was $94.04 \%$. Several international studies have documented the sensitivity of FNAC in thyroid nodules to range from $52-98 \% \cdot{ }^{17,18,21,22}$ Similarly, the international normal range is for specificity is 72 to $100 \%{ }^{17,22}$ and for PPV is 50 to $90 \%{ }^{17,22}$. The negative predictive value derived from this audit was $73.1 \%$.In a report of 19 studies and 20 series by Lewis et al 2009[2],the reported and re-calculated mean for negative predictivevalue was $84 \%$ and $93 \%$. So present study is fairly comparable to other studies.

\section{Conclusion}

FNAC is a simple, safe and cost-effective diagnostic modality in the investigation of thyroid disease with high specificity and accuracy. We concluded that FNAC diagnosis of malignancy is highly significant. A benign FNAC diagnosis should be viewed with caution as false negative results do occur and these patients should be followed up and any clinical suspicion of malignancy even in the presence of benign FNAC requires surgery. So, final diagnosis and treatment pattern should be based upon histopathology.

\section{References}

[1]. Burch HB, Burman KD, Reed HI, Buckner L, Raber T, Ownbey Jl. Fine needle aspiration of thyroid nodules. Determinants of insufficiency rate and malignancy yield at thyroidectomy. Acta Cytol 1996; 40: 1176-83.

[2]. Gharib H, Goellner JR. Fine-needle aspiration biopsy of the thyroid: an appraisal. Ann Intern Med 1993; 118: 282-9.

[3]. Franklyn JA, Sheppard MC. Aspiration cytology of thyroid. BMJ 1987; 295: 510-1.

[4]. Lowhagen T, Willems J, Lundell G, Sundblad R, Granberg P. Aspiration biopsy cytology in diagnosis of thyroid cancer. World J Surg .1981; 5: 61-73

[5]. Al-Sayer HM, Krukowski ZH, Williams VMM, Matheson NA. Fine needle aspiration cytology in isolated thyroid swellings: a prospective two year evaluation. BMJ 1985; 290: 1490-2.

[6]. Anderson JB, Webb AJ. Fine-needle aspiration biopsy and the diagnosis of thyroid cancer. Br J Surg 1987; 74: $292-6$.

[7]. Leonard N, Melcher DH. To operate or not to operate? The value of fine needle aspiration cytology in the assessment of thyroid swellings. J Clin Pathol 1997; 50: 941-3.

[8]. Gardner HA, Ducatman BS, Wang HH. Predictive value of fine-needle aspiration of the thyroid in the classification of follicular lesions. Cancer 1993; 71: 2598-603.

[9]. Miller JM, Kini SR, Hamburger JI. The diagnosis of malignant follicular neoplasms of the thyroid by needle biopsy. Cancer 1985; 55: 2812-7.

[10]. Suresh K, Shakil A, Abdullah D. Role of fine needle aspiration cytology in Thyroid diseases. Journal of Surgery Pakistan (International) 13 (1) January - March 2008.

[11]. Md. Shafiqul I, Belayat H S , Nasima A, Kazi S S, Mohammad A. Comparative study of FNAC and histopathology in the diagnosis of thyroid swelling. Bangladesh J Otorhinolaryngol. 16 (1); April 2010.

[12]. Lewis CM, Chang KP, Pitman M, WC Faquin , Randolph GW. Thyroid fine-needle aspiration biopsy: variability in reporting. Thyroid. 2009 Jul;19(7):717-23. doi: 10.1089/thy.2008.0425.Pubmed.

[13]. Monisha Choudhury, Smita Singh, Savita Agarwal. Diagnostic utility of ki67 and p53 immunostaining on solitary thyroid nodule A cytohistological and radionuclide scintigraphic study. Indian Journal Of Pathology \& Microbioology. 2011; 54 .3 : 472-475.

[14]. Pinki Pandey, Alok Dixit., Nanak C Mahajan. Fine-needle aspiration of the thyroid: A cytohistologic correlation with critical evaluation of discordant cases. Thyeroid Research and Practice.2012;9.2:32-39.

[15]. Mundasad Mcallidter I, Carson J (2006) Accuracy of fine needle aspiration cytology in diagnosis of thyroid swelling. Internet J Endocrinol 2(2):23-25.

[16]. Garib H, Papaini H, Valcavi R et all; AACE/AME Task Force in Thyroid Nodules. American Association of clinical Endocrinologist and Associazione Medici Endocrinologi medical guidelines for medical practice, diagnosis and management of thyroid nodules. Endocr Prati 2006;12: 63--102 